\title{
Cryoglobulinemic Glomerulonephritis
}

National Cancer Institute

\section{Source}

National Cancer Institute. Cryoglobulinemic Glomerulonephritis. NCI Thesaurus. Code C123050.

Glomerulonephritis in the context of cryog lobulinemia. 